Kasuistiken

Ophthalmologe 2022 $\cdot 119: 741-743$

https://doi.org/10.1007/s00347-021-01428-y

Eingegangen: 26. Januar 2021

Überarbeitet: 3. Mai 2021

Angenommen: 11. Mai 2021

Online publiziert: 9 . Juni 2021

(c) Der/die Autor(en) 2021

\section{Anamnese}

Ein 62-jähriger Patient wurde mit starken Schmerzen und einer zu dem Zeitpunkt seit 12 Tagen bestehenden Rötung und Schwellung der Augen notfallmäßig von seinem niedergelassenen Augenarzt an unsere Klinik überwiesen.

Die bisherige Behandlung durch den Hausaugenarzt umfasste Dexamethason, Neomycin und Polymyxin-B-Augentropfen 4-mal täglich, Perfluorhexyloctan-haltige Tränenersatzmittel 4-mal täglich sowie Retinolpalmitat Augensalbe zur Nacht.

Anamnestisch war eine weit fortgeschrittene Retinitis pigmentosa im Rahmen eines Refsum-Syndroms bei dem Patienten bekannt. Die Diagnose des Morbus Refsum war bereits in den 80er-Jahren gestellt worden. Eine Katarakt zeigte sich als Initialsymptom in den 70er-Jahren und wurde mit Vorderkammerlinsen in Kombination mit einer Iridektomie versorgt. Anschließend folgten die ersten Symptome der peripheren Neuropathie mit Gangschwierigkeiten. Symptome der neurosensorischen Hörminderung begannen in den 90er-Jahren. Eine kardiale Beteiligung des Morbus Refsum wurde verneint, regelmäßige kardiologische Kontrollen würden stattfinden. Nach Diagnosestellung erfolgten zunächst eine Plasmapherese und eine phytansäurearme Diät, diese hatte der Patient allerdings gegen ärztlichen Rat nicht mehr eingehalten.

\section{Klinischer Befund}

Der Visus am rechten Auge betrug Lichtschein, der Visus am linken Auge betrug Handbewegungen. In der

Isabel Weinstein (D) Fabian N. Fries - Marie Hartmann • Alaa Din Abdin • Berthold Seitz

Klinik für Augenheilkunde, Universitätsklinikum des Saarlandes UKS, Homburg/Saar, Deutschland

\title{
Ausgeprägte Bandkeratopathie bei Refsum-Syndrom
}

Spaltlampenbiomikroskopie imponierte eine ausgeprägte Bandkeratopathie mit Ablagerung von Kalkschollen und eine diffuse Hornhaut-Endothel-EpithelDekompensation rechts mehr als links (• Abb. 1). Die ausgeprägten Kalkschollen ließen sich auch in der optischen Kohärenztomographie (AS-OCT-CASIA 2; Tomey, Nagoya, Japan) eindeutig darstellen (-Abb. 2). Des Weiteren zeigten sich Vorderkammerlinsen, eine Iridektomie sowie eine Irisatrophie beidseits. Der Augeninnendruck lag applanatorisch gemessen bei $15 \mathrm{~mm} \mathrm{Hg}$ am rechten und bei $16 \mathrm{~mm} \mathrm{Hg}$ am linken Auge. Ein Funduseinblick war hornhautbedingt sowie aufgrund einer mangelnden Reaktion auf Mydriatika nicht möglich, allerdings zeigten sich die Netzhäute sonographisch anliegend. Die Goldmann-Perimetrie (Marke V/4) zeigte ein konzentrisch eingeschränktes Gesichtsfeld mit einem Restgesichtsfeld von weniger als $10 \mathrm{Grad}$ links (• Abb. 3), rechts war die Perimetrie visusbedingt nicht möglich. Das Ganzfeldelektroretinogramm (Ganzfeld-ERG, durchgeführt nach ISCEVStandard) zeigte die a- und b-Wellen skotopisch und in den Mischantworten

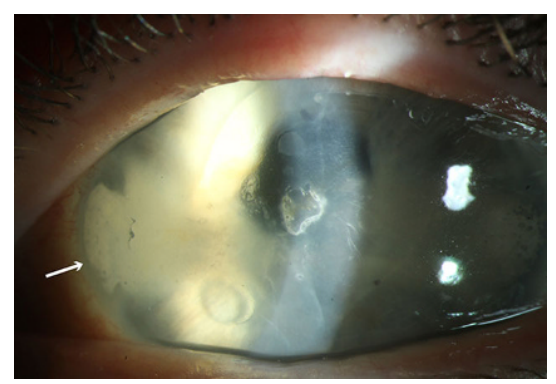

Abb. 1 ॥ Spaltlampenfoto des linken Auges mit ausgeprägter Bandkeratopathie und der Ablagerung von dichten Kalkschollen (Pfeil) fast erloschen rechts mehr als links sowie die photopische Antwort beidseits pathologisch reduziert.

Systemische Befunde des Patienten umfassten Cochleaimplantate, eine periphere Neuropathie und verkürzte Metatarsale beidseits (- Abb. 4). Der Phytansäurespiegel zeigte einen Wert von $214 \mathrm{mg} / \mathrm{l}$ (Referenzbereich < $5 \mathrm{mg} / \mathrm{l}$ ) im Rahmen eines Refsum-Syndroms.

\section{Therapie und Verlauf}

Es erfolgte eine Therapieumstellung auf phosphatfreie Präparate mit OfloxacinAugentropfen 5-mal täglich, Hyaluronsäure- und Perfluorhexyloctan-haltige Tränenersatzmitteln je 5-mal täglich sowie Retinolpalmitat-Augensalbe zur Nacht. Des Weiteren vereinbarten wir Termine (nach 4 Wochen am rechten sowie nach 16 Wochen am linken Auge) zur Durchführung einer Touchierung mit Ethylendiamintetraacetat (EDTA) in Kombination mit einer phototherapeutischen Keratektomie (PTK) (AMARIS 1050RS Excimerlaser, SCHWIND eyetech-solutions $\mathrm{GmbH}$, Kleinostheim, Germany) mit einem Durchmesser von $10 \mathrm{~mm}$ und einem Abtrag von $100 \mu \mathrm{m}$

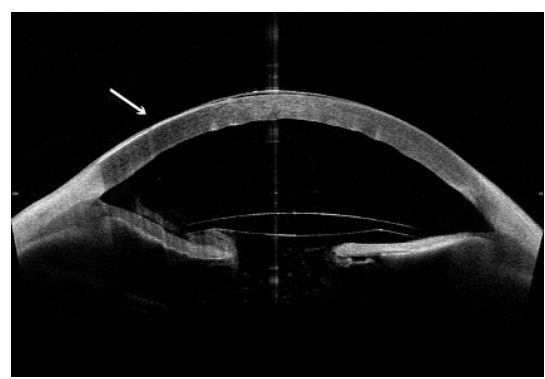

Abb. $2 \Delta$ Vorderabschnitt-OCT des linken Auges: dargestellt ist die Kalkscholle (Pfeil) im Rahmen der Bandkeratopathie 


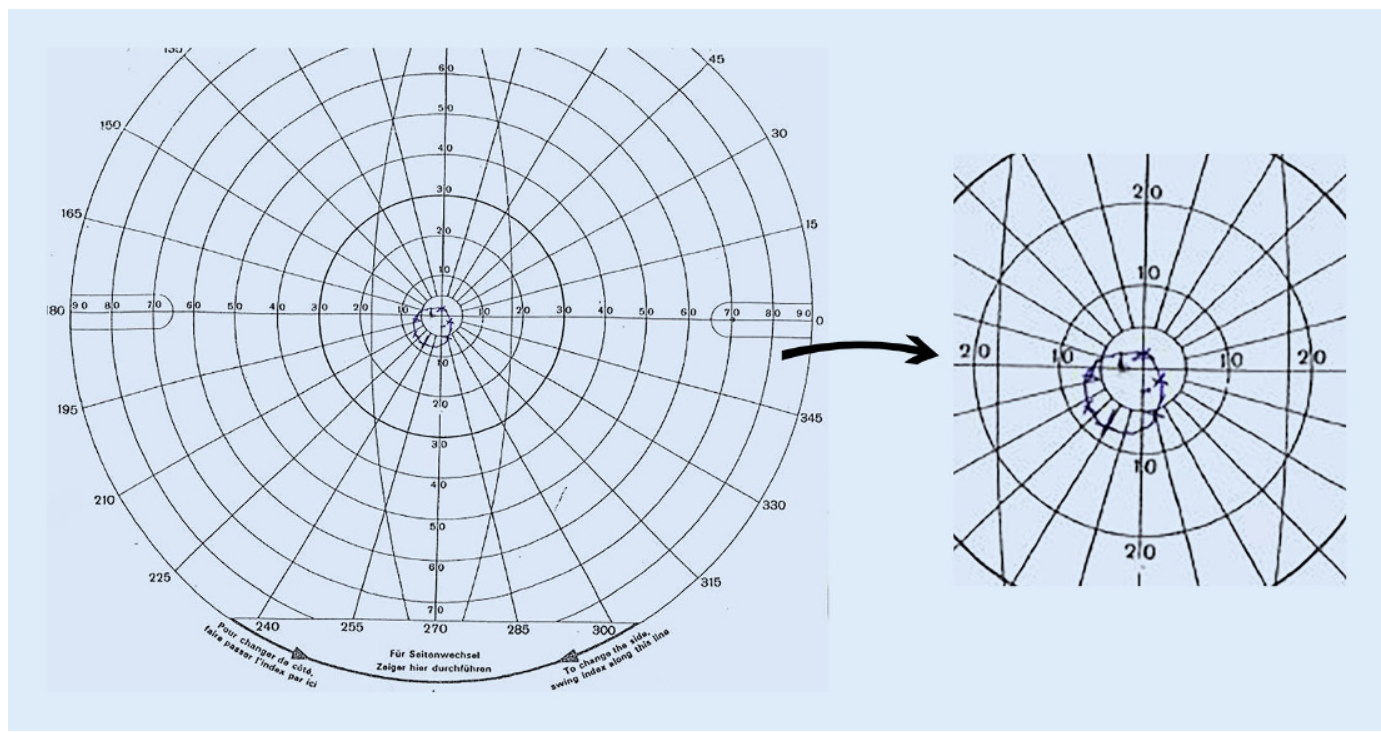

Abb. $3 \triangleleft$ Manuell durchgeführtes Goldmann Gesichtsfeld (Marke V/4) des linken Auges des Patienten mit einem konzentrisch eingeschränkten Restgesichtsfeld von unter $10 \mathrm{Grad}$

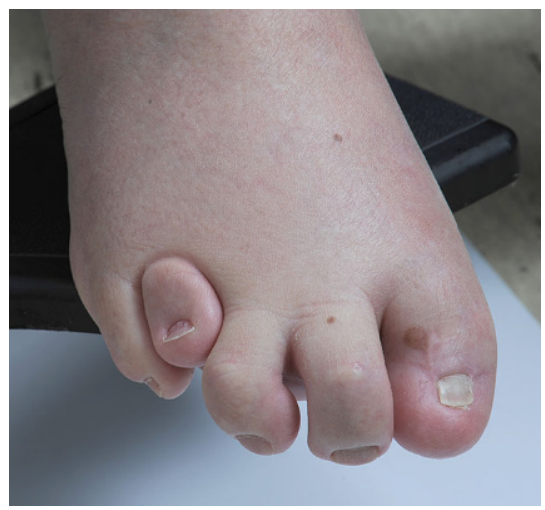

Abb. 4 \ Foto der verkürzten 4. Metatarsale

rechts sowie $50 \mu \mathrm{m}$ links jeweils ohne Maskierung, weil die verbleibende Oberfläche relativ glatt war.

Postoperativ zeigte sich spaltlampenbiomikroskopisch und in der Vorderabschnitt-OCT eine klare Hornhaut (- Abb. 5 und 6). In der Pachymetrie (AS-OCT-CASIA 2; Tomey, Nagoya, Japan) zeigte sich eine Abnahme der Hornhautdicke von $903 \mu \mathrm{m}$ präoperativ auf $719 \mu \mathrm{m}$ postoperativ am rechten sowie von $802 \mu \mathrm{m}$ präoperativ auf $601 \mu \mathrm{m}$ postoperativ am linken Auge. Darüber hinaus kam es am linken Auge zu einem Visusanstieg auf 0,1 cum correctione $\left(+6,50 /-1,75 / \mathrm{A} 104^{\circ}\right)$.

\section{Diskussion}

Das Refsum-Syndrom stellt eine seltene, autosomal-rezessiv vererbte motorischsensible Neuropathie dar, welche auf ei- ner Störung des Phytansäurestoffwechsels beruht. Sie ist nach ihrem Erstbeschreiber Sigvald Refsum benannt, welcher die Erkrankung erstmalig 1946 unter dem Namen „Heredopathia atactica polyneuritiformis" beschrieb [6]. Phytansäure, eine gesättigte, langkettige Fettsäure, wird ausschließlich über die Nahrung aufgenommen, kann durch einen Mangel an Phytanoyl-CoA-Hydroxylase nicht abgebaut werden und lagert sich folglich in Geweben ab [4, 10]. Die Prognose der Erkrankung ist unbehandelt schlecht, weshalb eine frühzeitige Diagnosestellung und eine strikte, phytansäurearme Diät zur Vermeidung von Erblindung, Taubheit und schweren neurologischen Komplikationen essenziell sind [3].

Eine atypische Retinitis pigmentosa (tapetoretinale Degeneration), ggf. mit Nachtblindheit und progressiver Gesichtsfeldverengung, ist bei Patienten mit Refsum-Syndrom in der Regel vorhanden und sollte v. a. in Zusammenhang mit verkürzten Metakarpalen und Metatarsalen differenzialdiagnostisch an eine Refsum-Erkrankung denken lassen.

Typische ophthalmologische Befunde schließen neben der Retinitis pigmentosa eine Irisatrophie, Katarakt, eine $\mathrm{Pu}-$ pillenverengung, eine verminderte $\mathrm{Pu}$ pillenreaktion auf Mydriatika sowie eine eingeschränkte Lichtreaktion ein [7, 8].

Weitere typische systemische Befunde umfassen eine periphere Polyneuropathie, neurosensorische Hörminderung, zerebelläre Ataxie, Herzrhythmusstörungen und Ichthyose.

Eine Ichthyose beschreibt eine meist genetisch hervorgerufene Verhornungsstörung der Haut. Diese kann autosomal-dominant, autosomal sowie X-chromosomal rezessiv vererbt werden und nichtsyndromal sowie syndromal auftreten. Die nichtsyndromale Ichthyose betrifft hauptsächlich die Haut, während bei syndromalen Ichthyosen auch weitere Organsysteme betroffen sein können. Das Refsum-Syndrom wird folglich zu der Gruppe der syndromalen Ichthyosen gezählt.

Es ist eine Vielzahl von Formen der Ichthyose bekannt, welchen verschiedene Defekte und somit verschiedene Pathomechanismen zugrunde liegen. In gesunder Haut sind Keratinozyten durch Zellkontakte verbunden und werden bei Abschuppung durch Enzyme abgebaut. An der Bildung der normalen Hautbarriere sind Proteine des Lipidstoffwechsels beteiligt. Eine Fehlaktivität dieser führt zu einer abnormalen Differenzierung und Proliferation der Epidermis. Charakteristischerweise zeigen sich eine Hyperkeratose und Schuppung [9].

Anton-Lambrecht und Kahlke setzten sich in einer Publikation von 1974 ausgiebig mit dem Pathomechanismus der Ichthyose der Refsum-Erkrankung auseinander und konnten auf eine Korrelation zwischen der Verhornungsstörung beim Refsum-Syndrom und der Ablagerung der Phytansäure schließen [1]. 


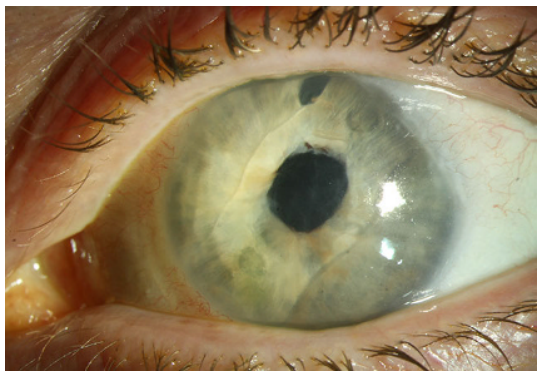

Abb. 5 ॥ Spaltlampenfoto des linken Auges mit postoperativ klarer Hornhaut. Intraokulär sind die Vorderkammerlinse sowie die Iridektomie sichtbar

Bereits 1967 befassten sich Jay et al. mit der okulären Manifestation einer Ichthyose. Sie konnten epitheliale sowie tiefstromale Veränderungen bei Patienten mit Ichthyose nachweisen und berichten über einen Patienten mit einer beginnenden Bandkeratopathie im Rahmen einer Ichthyosis vulgaris [5].

Während in einer Publikation von Baum et al. eine bullöse Keratopathie bei Patienten mit Refsum-Syndrom bereits beschrieben wurde, gibt es nach unserem besten Wissen bisher keinen Fallbericht eines Patienten mit Bandkeratopathie und Ablagerung von Kalkschollen als Manifestation einer Ichthyose im Rahmen eines Refsum-Syndroms [2].

Eine zielführende Behandlungsoption stellt die EDTA-Touchierung in Kombination mit einer PTK dar, um sowohl die Kalkschollen zu entfernen, Schmerzen $\mathrm{zu}$ lindern und einen Visusanstieg $\mathrm{zu}$ erzielen.

\section{Fazit für die Praxis}

- Das Refsum-Syndrom ist eine seltene, autosomal-rezessiv vererbte Stoffwechselerkrankung, welche auf einer Störung des Phytansäurestoffwechsels beruht.

- Typische ophthalmologische Befunde schließen eine Retinitis pigmentosa, eine Irisatrophie und Katarakt ein, typische systemische Befunde umfassen eine periphere Polyneuropathie, neurosensorische Hörminderung, zerebelläre Ataxie, Herzrhythmusstörungen und Ichthyose.

- Als Manifestation einer Ichthyose kann es beim Refsum-Syndrom zu einer Bandkeratopathie mit Ablage-

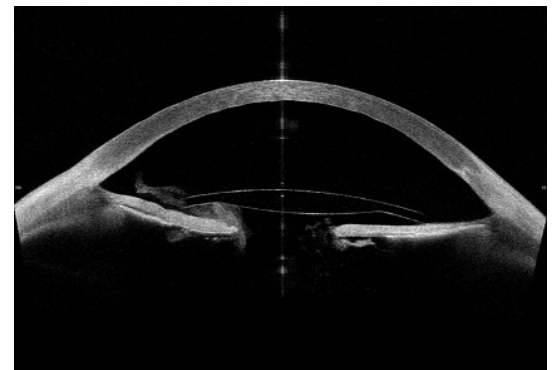

Abb. $6 \Delta$ Vorderabschnitt-OCT des linken Auges postoperativ: dargestellt ist eine klare Hornhaut ohne Kalkablagerung

rung von Kalkschollen und einem dadurch noch weiter reduzierten Visus bis hin zu stechenden Schmerzen kommen.

- Die EDTA-Touchierung in Kombination mit einer PTK stellt eine zielführende Behandlungsoption dar.

\section{Korrespondenzadresse

Isabel Weinstein
Klinik für Augenheilkunde,
Universitätsklinikum des
Saarlandes UKS
Kirrberger Str. 100,
66421 Homburg/Saar,
Deutschland
isabel.weinstein@uks.eu

Funding. Open Access funding enabled and organized by Projekt DEAL.

\section{Einhaltung ethischer Richtlinien}

Interessenkonflikt. I. Weinstein, F.N. Fries, M. Hartmann, A.D. Abdin und B. Seitz geben an, dass kein Interessenkonflikt besteht.

Für diesen Beitrag wurden von den Autoren keine Studien an Menschen oder Tieren durchgeführt. Für die aufgeführten Studien gelten die jeweils dort angegebenen ethischen Richtlinien. Für Bildmaterial oder anderweitige Angaben innerhalb des Manuskripts, über die Patienten zu identifizieren sind, liegt von ihnen und/oder ihren gesetzlichen Vertretern eine schriftliche Einwilligung vor.

Open Access. Dieser Artikel wird unter der Creative Commons Namensnennung 4.0 International Lizenz veröffentlicht, welche die Nutzung, Vervielfältigung, Bearbeitung, Verbreitung und Wiedergabe in jeglichem Medium und Format erlaubt, sofern Sie den/die ursprünglichen Autor(en) und die Quelle ordnungsgemäßnennen, einen Link zur Creative Commons Lizenz beifügen und angeben, ob Änderungen vorgenommen wurden.
Die in diesem Artikel enthaltenen Bilder und sonstiges Drittmaterial unterliegen ebenfalls der genannten Creative Commons Lizenz, sofern sich aus der Abbildungslegende nichts anderes ergibt. Sofern das betreffende Material nicht unter der genannten Creative Commons Lizenz steht und die betreffende Handlung nicht nach gesetzlichen Vorschriften erlaubt ist, ist für die oben aufgeführten Weiterverwendungen des Materials die Einwilligung des jeweiligen Rechteinhabers einzuholen.

Weitere Details zur Lizenz entnehmen Sie bitte der Lizenzinformation auf http://creativecommons.org/ licenses/by/4.0/deed.de.

\section{Literatur}

1. Anton-Lamprecht I, Kahlke W (1974) Zur UItrastruktur hereditärer Verhornungsstörungen, $\mathrm{V}$, Ichthyosis beim Refsum-Syndrom. Arch Derm Forsch 250:185-206

2. Baum JL, Tannenbaum M, Kolodny EH (1965) Refsum's syndrome with corneal involvement. Am JOphthalmol 60(4):699-708

3. Eldjarn L, TryKetal (1966) Dietary effects on serumphytanic-acid levels and on clinical manifestations in heredopathia atactica polyneuritiformis. Lancet 1:691-693

4. Jansen GA, Ofman R et al (1997) Refsum disease is caused by mutations in the phytanoyl-CoA hydroxylase gene. Nat Genet 17:190-193

5. Jay B, Blach RKet al (1968) Ocular manifestations of ichthyosis. Br JOphthalmol 52(3):217-226. https:// doi.org/10.1136/bjo.52.3.217

6. Refsum S (1946) Heredopathia atactica polyneuritiformis. Acta Psychiatr Scand 38:1

7. Richterich R, Moser Het al (1965) Refsum's disease (Heredopathia atactica polyneuritiformis). Hum Genet 1(4):322-332. https://doi.org/10.1007/ BF00273974

8. Rüther K (2005) Die adulte Refsum-Erkrankung. Ophthalmologe 102:772-777

9. Süßmuth K, Traupe H, Metze D, Oji V (2020) Ichthyosen im klinischen Alltag: Umgang mit einer seltenen Erkrankungsgruppe. J Dtsch Dermatol Ges 18(3):225-245

10. Wierzbicki AS, Lloyd MD et al (2002) Refsum's disease: a peroxisomal disorder affecting phytanic acid a-oxidation. J Neurochem 80:727-735 